\title{
Approximation properties of bivariate extension of $q$-Szász-Mirakjan-Kantorovich operators
}

\author{
Mediha Örkcü* \\ Dedicated to Professor Hari M Srivastava
}

${ }^{*}$ Correspondence:

medihaakcay@gazi.edu.tr

Department of Mathematics,

Faculty of Sciences, Teknik-okullar,

Gazi University, Ankara, 06500,

Turkey

\begin{abstract}
In the present paper, a bivariate generalization of the q-Szász-Mirakjan-Kantorovich operators is constructed by $q_{R}$-integral and these operators' weighted A-statistical approximation properties are established. Also, we estimate the rate of pointwise convergence of the proposed operators by modulus of continuity.

MSC: $41 \mathrm{~A} 25 ; 41 \mathrm{~A} 36$
\end{abstract}

Keywords: bivariate operators; weighted A-statistical approximation; Szász-Mirakjan operators; Kantorovich-type operators; q-integers

\section{Introduction}

In [1] the Kantorovich type generalization of the Szász-Mirakjan operators is defined by

$$
K_{n}(f, x):=n e^{-n x} \sum_{k=0}^{\infty} \frac{(n x)^{k}}{k !} \int_{k / n}^{(k+1) / n} f(t) d t, \quad f \in C[0, \infty), 0 \leq x<\infty .
$$

In [2], for each positive integer $n$, Aral and Gupta defined q-type generalization of SzászMirakjan operators as

$$
S_{n}^{q}(f ; x):=E_{q}\left(-[n]_{q} \frac{x}{b_{n}}\right) \sum_{k=0}^{\infty} f\left(\frac{[k]_{q} b_{n}}{[n]_{q}}\right) \frac{\left([n]_{q} x\right)^{k}}{[k]_{q} !\left(b_{n}\right)^{k}}
$$

where $0<q<1, f \in C[0, \infty), 0 \leq x<\frac{b_{n}}{(1-q)[n]_{q}}, b_{n}$ is a sequence of positive numbers such that $\lim _{x \rightarrow \infty} b_{n}=\infty$.

Recently, $q$-type generalization of Szász-Mirakjan operators, which was different from that in [2], was introduced, and the convergence properties of these operators were studied by Mahmudov [3]. Weighted statistical approximation properties of the modified $q$-SzászMirakjan operators were obtained in [4]. Also, Durrmeyer and Kantorovich-type generalizations of the linear positive operators based on $q$-integers were studied by some authors. The Bernstein-Durrmeyer operators related to the $q$-Bernstein operators were studied by Derriennic [5]. Gupta [6] introduced and studied approximation properties of $q$-Durrmeyer operators. The generalizations of the $q$-Baskakov-Kantorovich operators 
were constructed and weighted statistical approximation properties of these operators were examined in [7] and [8]. The $q$-extensions of the Szász-Mirakjan, Szász-MirakjanKantorovich, Szász-Schurer and Szász-Schurer-Kantorovich operators were given shortly in [8]. Generalized Szász Durrmeyer operators were studied in [9]. With the help of $q_{R}$-integral, Örkcü and Doğru [10] introduced a Kantorovich-type modification of the $q$-Szász-Mirakjan operators as follows:

$$
K_{n}^{q}(f ; x):=[n]_{q} E_{q}\left(-[n]_{q} \frac{x}{q}\right) \sum_{k=0}^{\infty} \frac{\left([n]_{q} x\right)^{k}}{[k]_{q} ! q^{k}} \int_{q[k]_{q} /[n]_{q}}^{[k+1]_{q} /[n]_{q}} f(t) d_{q}^{R} t
$$

where $q \in(0,1), 0 \leq x<\frac{q}{1-q^{n}}, f \in C[0, \infty)$.

The paper of Mursaleen et al. [11] is one of the latest references on approximation by $q$-analogue. They investigated approximation properties for new $q$-Lagrange polynomials. Also, the $q$-analogue of Bernstein-Schurer-Stancu operators were introduced in [12].

On the other hand, Stancu [13] first introduced linear positive operators in two and several dimensional variables. Afterward, Barbosu [14] introduced a generalization of two-dimensional Bernstein operators based on $q$-integers and called them bivariate $q$-Bernstein operators. In recent years, many results have been obtained in the Korovkintype approximation theory via $A$-statistical convergence for functions of several variables (for instance, [15-17]).

In this study, we construct a bivariate generalization of the Szász-Mirakjan-Kantorovich operators based on $q$-integers and obtain the weighted $A$-statistical approximation properties of these operators.

Now we recall some definitions about $q$-integers. For any non-negative integer $r$, the $q$-integer of the number $r$ is defined by

$$
[r]_{q}= \begin{cases}1+q+\cdots+q^{r-1} & \text { if } q \neq 1, \\ r & \text { if } q=1\end{cases}
$$

where $q$ is a positive real number. The $q$-factorial is defined as

$$
[r]_{q} != \begin{cases}{[1]_{q}[2]_{q} \cdots[r]_{q}} & \text { if } r=1,2, \ldots \\ 1 & \text { if } r=0 .\end{cases}
$$

Two $q$-analogues of the exponential function $e^{x}$ are given as

$$
\begin{aligned}
& E_{q}(x)=\sum_{n=0}^{\infty} q^{n(n-1) / 2} \frac{x^{n}}{[n]_{q} !}, \quad x \in \mathbb{R}, \\
& \varepsilon_{q}(x)=\sum_{n=0}^{\infty} \frac{x^{n}}{[n]_{q} !}, \quad|x|<\frac{1}{1-q} .
\end{aligned}
$$

The following relation between $q$-exponential functions $E_{q}(x)$ and $\varepsilon_{q}(x)$ holds

$$
E_{q}(x) \varepsilon_{q}(-x)=1, \quad|x|<\frac{1}{1-q} .
$$


In the fundamental books about $q$-calculus (see $[18,19]$ ), the $q$-integral of the function $f$ over the interval $[0, b]$ is defined by

$$
\int_{0}^{b} f(t) d_{q} t=b(1-q) \sum_{j=0}^{\infty} f\left(b q^{j}\right) q^{j}, \quad 0<q<1 .
$$

If $f$ is integrable over $[0, b]$, then

$$
\lim _{q \rightarrow 1^{-}} \int_{0}^{b} f(t) d_{q} t=\int_{0}^{b} f(t) d t .
$$

A generally accepted definition for $q$-integral over an interval $[a, b]$ is

$$
\int_{a}^{b} f(t) d_{q} t=\int_{0}^{b} f(t) d_{q} t-\int_{0}^{a} f(t) d_{q} t
$$

In order to generalize and spread the existing inequalities, Marinkovic et al. considered a new type of $q$-integral. So, the problems that ensue from the general definition of $q$-integral were overcome. The Riemann-type $q$-integral $[20]$ in the interval $[a, b]$ was defined as

$$
\int_{a}^{b} f(t) d_{q}^{R} t=(1-q)(b-a) \sum_{j=0}^{\infty} f\left(a+(b-a) q^{j}\right) q^{j}, \quad 0<q<1 .
$$

This definition includes only a point inside the interval of the integration.

\section{Construction of the bivariate operators}

The aim of this part is to construct a bivariate extension of the operators defined by (1.1).

For $n \in \mathbb{N}, 0<q_{1}, q_{2}<1$ and $0 \leq x<\frac{q_{1}}{1-q_{1}^{n}}, 0 \leq y<\frac{q_{2}}{1-q_{2}^{n}}$, the bivariate extension of the operators $K_{n}^{q}(f ; x)$ is as follows:

$$
\begin{aligned}
K_{n}^{q_{1}, q_{2}}(f ; x, y)= & {[n]_{q_{1}}[n]_{q_{2}} E_{q_{1}}\left(-[n]_{q_{1}} \frac{x}{q_{1}}\right) E_{q_{2}}\left(-[n]_{q_{2}} \frac{y}{q_{2}}\right) } \\
& \times \sum_{l=0}^{\infty} \sum_{k=0}^{\infty} \frac{\left([n]_{q_{1}} x\right)^{k}}{\left[[k]_{q_{1}} ! q_{1}^{k}\right.} \frac{\left([n]_{q_{2}} y\right)^{l}}{[l]_{q_{2}} ! q_{2}^{l}} \\
& \times \int_{q_{2}[l]_{q_{2}} /[n]_{q_{2}}}^{[l+1]_{q_{2}} /[n]_{q_{2}}} \int_{\left.q_{1}[k]\right]_{q_{1}}\left[[n]_{q_{1}}\right.}^{[k+1]_{q_{1}}\left[[n]_{q_{1}}\right.} f(t, s) d_{q_{1}}^{R} t d_{q_{2}}^{R} s,
\end{aligned}
$$

where

$$
\begin{aligned}
\int_{c}^{d} \int_{a}^{b} f(t, s) d_{q_{1}}^{R} t d_{q_{2}}^{R} s= & \left(1-q_{1}\right)\left(1-q_{2}\right)(b-a)(c-d) \\
& \times \sum_{j=0}^{\infty} \sum_{i=0}^{\infty} f\left(a+(b-a) q_{1}^{i}, c+(c-d) q_{2}^{j}\right) q_{1}^{i} q_{2}^{j} .
\end{aligned}
$$

Also, $f$ is a $q_{R}$-integrable function, so the series in (2.2) converges. It is clear that the operators given in (2.1) are linear and positive.

First, let us give the following lemma. 
Lemma 1 Let $e_{i j}=x^{i} y^{j},(i, j) \in \mathbb{N}_{0} \times \mathbb{N}_{0}$ with $i+j \leq 2$ be the two-dimensional test functions.

Then the following results hold for the operators given by (2.1):

(i) $\quad K_{n}^{q_{1}, q_{2}}\left(e_{00} ; x, y\right)=1$;

(ii) $K_{n}^{q_{1}, q_{2}}\left(e_{10} ; x, y\right)=x+\frac{1}{[2]_{q_{1}}} \frac{1}{[n]_{q_{1}}}$;

(iii) $K_{n}^{q_{1}, q_{2}}\left(e_{01} ; x, y\right)=y+\frac{1}{[2]_{q_{2}}} \frac{1}{[n]_{q_{2}}}$;

(iv) $K_{n}^{q_{1}, q_{2}}\left(e_{20} ; x, y\right)=q_{1} x^{2}+\left(q_{1}+\frac{2}{[2]_{q_{1}}}\right) \frac{1}{[n]_{q_{1}}} x+\frac{1}{[3]_{q_{1}}} \frac{1}{[n]_{q_{1}}^{2}}$;

(v) $K_{n}^{q_{1}, q_{2}}\left(e_{02} ; x, y\right)=q_{2} y^{2}+\left(q_{2}+\frac{2}{[2]_{q_{2}}}\right) \frac{1}{[n]_{q_{2}}} y+\frac{1}{[3]_{q_{2}}} \frac{1}{[n]_{q_{2}}^{2}}$.

Proof From $\int_{q_{2}[l] q_{2} /[n]_{q_{2}}}^{\left.[l+1] q_{q_{2}} /[n]\right]_{q_{2}}} \int_{q_{1}[k] q_{q_{1}} /[n] q_{q_{1}}}^{[k+1]} d_{q_{1}}^{R} t d_{q_{2}}^{R} s=\frac{1}{[n]_{q_{1}}[n]_{q_{2}}}$ and the equality in (1.2), we have

$$
\begin{aligned}
K_{n}^{q_{1}, q_{2}}\left(e_{00} ; x, y\right)= & E_{q_{1}}\left(-[n]_{q_{1}} \frac{x}{q_{1}}\right) E_{q_{2}}\left(-[n]_{q_{2}} \frac{y}{q_{2}}\right) \\
& \times \sum_{l=0}^{\infty} \sum_{k=0}^{\infty} \frac{\left([n]_{q_{1}} x\right)^{k}}{[k]_{q_{1}} ! q_{1}^{k}} \frac{\left([n]_{q_{2}} y\right)^{l}}{[l]_{q_{2}} ! q_{2}^{l}} \\
= & 1 .
\end{aligned}
$$

Since $\int_{q_{2}[l] q_{2} /[n] q_{q_{2}}}^{[l+1]]_{q_{1}}\left[[n] q_{2}\right.} \int_{q_{1}[k] q_{1} /[n] q_{q_{1}}}^{[k+1] q_{q_{1}} /\left[[]_{q_{1}}\right.} t d_{q_{1}}^{R} t d_{q_{2}}^{R} s=\frac{1}{[n]]_{q_{1}}[n] q_{q_{2}}}\left(\frac{q_{1}[k] q_{q_{1}}}{[n]]_{q_{1}}}+\frac{1}{[2] q_{1}} \frac{1}{[n] q_{q_{1}}}\right)$, we get from the linearity of $K_{n}^{q_{1}, q_{2}}$ that

$$
\begin{aligned}
K_{n}^{q_{1}, q_{2}}\left(e_{10} ; x, y\right)= & E_{q_{1}}\left(-[n]_{q_{1}} \frac{x}{q_{1}}\right) E_{q_{2}}\left(-[n]_{q_{2}} \frac{y}{q_{2}}\right) \\
& \times \sum_{l=0}^{\infty} \sum_{k=0}^{\infty} \frac{q_{1}[k]_{q_{1}}}{[n]_{q_{1}}} \frac{\left([n]_{q_{1}} x\right)^{k}}{[k]_{q_{1}} ! q_{1}^{k}} \frac{\left([n]_{q_{2}} y\right)^{l}}{[l]_{q_{2}} ! q_{2}^{l}} \\
& +E_{q_{1}}\left(-[n]_{q_{1}} \frac{x}{q_{1}}\right) E_{q_{2}}\left(-[n]_{q_{2}} \frac{y}{q_{2}}\right) \\
& \times \sum_{l=0}^{\infty} \sum_{k=0}^{\infty} \frac{1}{[2]_{q_{1}}} \frac{1}{[n]_{q_{1}}} \frac{\left([n]_{q_{1}} x\right)^{k}}{[k]_{q_{1}} ! q_{1}^{k}} \frac{\left([n]_{q_{2}} y\right)^{l}}{[l]_{q_{2}} ! q_{2}^{l}}
\end{aligned}
$$

Then we have from the definition of $q$-factorial and $K_{n}^{q_{1}, q_{2}}\left(e_{00} ; x, y\right)=1$

$$
\begin{aligned}
K_{n}^{q_{1}, q_{2}}\left(e_{10} ; x, y\right)= & E_{q_{1}}\left(-[n]_{q_{1}} \frac{x}{q_{1}}\right) E_{q_{2}}\left(-[n]_{q_{2}} \frac{y}{q_{2}}\right) \\
& \times \sum_{l=0}^{\infty} \sum_{k=1}^{\infty} \frac{x\left([n]_{q_{1}} x\right)^{k-1}}{[k-1]_{q_{1}} ! q_{1}^{k-1}} \frac{\left([n]_{q_{2}} y\right)^{l}}{[l]_{q_{2}} ! q_{2}^{l}}+\frac{1}{[2]_{q_{1}}} \frac{1}{[n]_{q_{1}}} \\
= & x+\frac{1}{[2]_{q_{1}}} \frac{1}{[n]_{q_{1}}} .
\end{aligned}
$$


Similarly, we write that

$$
K_{n}^{q_{1}, q_{2}}\left(e_{01} ; x, y\right)=y+\frac{1}{[2]_{q_{2}}} \frac{1}{[n]_{q_{2}}} .
$$

Now we compute the value $K_{n}^{q_{1}, q_{2}}\left(e_{20} ; x, y\right)$. Applying the equalities $\int_{\left.q_{2}[l]\right]_{q_{2}} /[n]_{q_{2}}}^{[l+1]_{q_{2}} /[n]_{2}} \times$ $\int_{q_{1}[k]_{q_{1}} /[n]_{q_{1}}}^{[k+1]_{q_{1}} /[n]_{q_{1}}} t^{2} d_{q_{1}}^{R} t d_{q_{2}}^{R} s=\frac{1}{[n]_{q_{1}}[n]_{q_{2}}}\left(\frac{q_{1}^{2}[k]_{q_{1}}^{2}}{[n]_{q_{1}}^{2}}+\frac{1}{[2]_{q_{1}}} \frac{2 q_{1}[k]_{q_{1}}}{[n]_{q_{1}}^{2}}+\frac{1}{[3]_{q_{1}}} \frac{1}{[n]_{q_{1}}^{2}}\right), K_{n}^{q_{1}, q_{2}}\left(e_{10} ; x, y\right)=x+$ $\frac{1}{[2]_{q_{1}}} \frac{1}{[n]_{q_{1}}}$ and $K_{n}^{q_{1}, q_{2}}\left(e_{00} ; x, y\right)=1$, we obtain

$$
\begin{aligned}
K_{n}^{q_{1}, q_{2}}\left(e_{20} ; x, y\right)= & E_{q_{1}}\left(-[n]_{q_{1}} \frac{x}{q_{1}}\right) E_{q_{2}}\left(-[n]_{q_{2}} \frac{y}{q_{2}}\right) \\
& \times \sum_{l=0}^{\infty} \sum_{k=0}^{\infty} \frac{q_{1}^{2}[k]_{q_{1}}^{2}}{[n]_{q_{1}}^{2}} \frac{\left([n]_{q_{1}} x\right)^{k}}{[k]_{q_{1}} ! q_{1}^{k}} \frac{\left([n]_{q_{2}} y\right)^{l}}{[l]_{q_{2}} ! q_{2}^{l}} \\
& +\frac{2}{[2]_{q_{1}}} \frac{1}{[n]_{q_{1}}} x+\frac{1}{[3]_{q_{1}}} \frac{1}{[n]_{q_{1}}^{2}} .
\end{aligned}
$$

Next, using the fact that $[k]_{q_{1}}=q_{1}[k-1]_{q_{1}}+1$, we obtain

$$
\begin{aligned}
& K_{n}^{q_{1}, q_{2}}\left(e_{20} ; x, y\right)= E_{q_{1}}\left(-[n]_{q_{1}} \frac{x}{q_{1}}\right) E_{q_{2}}\left(-[n]_{q_{2}} \frac{y}{q_{2}}\right) \\
& \times \sum_{l=0}^{\infty} \sum_{k=1}^{\infty} \frac{q_{1}[k-1]_{q_{1}}+1}{[n]_{q_{1}}^{2}} \frac{\left([n]_{q_{1}} x\right)^{k}}{[k-1]_{q_{1}} ! q_{1}^{k-2}} \frac{\left([n]_{q_{2}} y\right)^{l}}{[l]_{q_{2}} ! q_{2}^{l}} \\
&+\frac{2}{[2]_{q_{1}}} \frac{1}{[n]_{q_{1}}} x+\frac{1}{[3]_{q_{1}}} \frac{1}{[n]_{q_{1}}^{2}} \\
&= E_{q_{1}}\left(-[n]_{q_{1}} \frac{x}{q_{1}}\right) E_{q_{2}}\left(-[n]_{q_{2}} \frac{y}{q_{2}}\right) \\
& \times \sum_{l=0}^{\infty} \sum_{k=2}^{\infty} \frac{q_{1} x^{2} \frac{\left([n]_{q_{1}} x\right)^{k-2}}{[k-2]_{q_{1}} ! q_{1}^{k-2}} \frac{\left([n]_{q_{2}} y\right)^{l}}{[l]_{q_{2}} ! q_{2}^{l}}}{} \\
&+E_{q_{1}}\left(-[n]_{q_{1}} \frac{x}{q_{1}}\right) E_{q_{2}}\left(-[n]_{q_{2}} \frac{y}{q_{2}}\right) \\
& \times \sum_{l=0}^{\infty} \sum_{k=1}^{\infty} \frac{q_{1}}{[n]_{q_{1}}} x \frac{\left([n]_{q_{1}} x\right)^{k-1}}{[k-1]_{q_{1}} ! q_{1}^{k-1}} \frac{\left([n]_{q_{2}} y\right)^{l}}{[l]_{q_{2}} ! q_{2}^{l}} \\
&+\frac{2}{[2]_{q_{1}}} \frac{1}{[n]_{q_{1}}} x+\frac{1}{[3]_{q_{1}}} \frac{1}{[n]_{q_{1}}^{2}} \\
& x^{2}+\left(q_{1}+\frac{2}{[2]_{q_{1}}}\right) \frac{1}{[n]_{q_{1}}} x+\frac{1}{[3]_{q_{1}}} \frac{1}{[n]_{q_{1}}^{2}} .
\end{aligned}
$$

Similarly, we write

$$
K_{n}^{q_{1}, q_{2}}\left(e_{02} ; x, y\right)=q_{2} y^{2}+\left(q_{2}+\frac{2}{[2]_{q_{2}}}\right) \frac{1}{[n]_{q_{2}}} y+\frac{1}{[3]_{q_{2}}} \frac{1}{[n]_{q_{2}}^{2}}
$$

which completes the proof. 


\section{A-Statistical approximation properties}

The Korovkin-type theorem for functions of two variables was proved by Volkov [21]. The theorem on weighted approximation for functions of several variables was proved by Gadjiev in [22].

Let $B_{\omega}$ be the space of real-valued functions defined on $\mathbb{R}^{2}$ and satisfying the bounded condition $|f(x, y)| \leq M_{f} \omega(x, y)$, where $\omega(x, y) \geq 1$ for all $(x, y) \in \mathbb{R}^{2}$ is called a weight function if it is continuous on $\mathbb{R}^{2}$ and $\lim _{\sqrt{x^{2}+y^{2}} \rightarrow \infty} \omega(x, y)=\infty$. We denote by $C_{\omega}$ the space of all continuous functions in the $B_{\omega}$ with the norm

$$
\|f\|_{\omega}=\sup _{(x, y) \in \mathbb{R}^{2}} \frac{|f(x, y)|}{\omega(x, y)} .
$$

Theorem 1 [22] Let $\omega_{1}(x, y)$ and $\omega_{2}(x, y)$ be weight functions satisfying

$$
\lim _{\sqrt{x^{2}+y^{2}} \rightarrow \infty} \frac{\omega_{1}(x, y)}{\omega_{2}(x, y)}=0
$$

Assume that $T_{n}$ is a sequence of linear positive operators acting from $C_{\omega_{1}}$ to $B_{\omega_{2}}$. Then, for all $f \in C_{\omega_{1}}$,

$$
\lim _{n \rightarrow \infty}\left\|T_{n} f-f\right\|_{\omega_{2}}=0
$$

if and only if

$$
\lim _{n \rightarrow \infty}\left\|T_{n} F_{v}-F_{v}\right\|_{\omega_{1}}=0 \quad(v=0,1,2,3),
$$

where $F_{0}(x, y)=\frac{\omega_{1}(x)}{1+x^{2}+y^{2}}, F_{1}(x, y)=\frac{x \omega_{1}(x)}{1+x^{2}+y^{2}}, F_{2}(x, y)=\frac{y \omega_{1}(x)}{1+x^{2}+y^{2}}, F_{3}(x, y)=\frac{\left(x^{2}+y^{2}\right) \omega_{1}(x)}{1+x^{2}+y^{2}}$.

In [16], using the concept of $A$-statistical convergence, Erkuş and Duman investigated a Korovkin-type approximation result for a sequence of positive linear operators defined on the space of all continuous real-valued functions on any compact subset of the real $m$-dimensional space.

Now we recall the concepts of regularity of a summability matrix and $A$-statistical convergence. Let $A:=\left(a_{n k}\right)$ be an infinite summability matrix. For a given sequence $x:=\left(x_{k}\right)$, the $A$-transform of $x$, denoted by $A x:=\left((A x)_{n}\right)$, is defined as $(A x)_{n}:=\sum_{k=1}^{\infty} a_{n k} x_{k}$ provided the series converges for each $n$. $A$ is said to be regular if $\lim _{n}(A x)_{n}=L$ whenever $\lim x=L$ [23]. Suppose that $A$ is a non-negative regular summability matrix. Then $x$ is $A$-statistically convergent to $L$ if for every $\varepsilon>0, \lim _{n} \sum_{k:\left|x_{k}-L\right| \geq \varepsilon} a_{n k}=0$, and we write $s t_{A}-\lim x=L$ [24]. Actually, $x$ is $A$-statistically convergent to $L$ if and only if, for every $\varepsilon>0, \delta_{A}\left(k \in \mathbb{N}:\left|x_{k}-L\right| \geq \varepsilon\right)=0$, where $\delta_{A}(K)$ denotes the $A$-density of the subset $K$ of the natural numbers and is given by $\delta_{A}(K):=\lim _{n} \sum_{k=1}^{\infty} a_{n k} \chi_{K}(k)$ provided the limit exists, where $\chi_{K}$ is the characteristic function of $K$. If $A=C_{1}$, the Cesáro matrix of order one, then $A$-statistical convergence reduces to the statistical convergence [25]. Also, taking $A=I$, the identity matrix, $A$-statistical convergence coincides with the ordinary convergence. 
We consider $\omega_{1}(x, y)=1+x^{2}+y^{2}$ and $\omega_{2}(x, y)=\left(1+x^{2}+y^{2}\right)^{1+\alpha}$ for $\alpha>0,(x, y) \in \mathbb{R}_{0}^{2}$, where $\mathbb{R}_{0}^{2}:=\left\{(x, y) \in \mathbb{R}^{2}: x \geq 0, y \geq 0\right\}$.

We obtain statistical approximation properties of the operator defined by (2.1) with the help of Korovkin-type theorem given in [26]. Let $\left(q_{1, n}\right)$ and $\left(q_{2, n}\right)$ be two sequences in the interval $(0,1)$ so that

$$
\begin{aligned}
& s t_{A}-\lim _{n} q_{1, n}^{n}=1 \quad \text { and } \quad s t_{A}-\lim _{n} q_{2, n}^{n}=1, \\
& s t_{A}-\lim _{n} \frac{1}{[n]_{q_{1, n}}}=0 \quad \text { and } \quad s t_{A}-\lim _{n} \frac{1}{[n]_{q_{2, n}}}=0 .
\end{aligned}
$$

Theorem 2 Let $A=\left(a_{n k}\right)$ be a nonnegative regular summability matrix, and let $\left(q_{1, n}\right)$ and $\left(q_{2, n}\right)$ be two sequences satisfying (3.1). Then, for any function $f \in C_{\omega_{1}}\left(\mathbb{R}_{0}^{2}\right)$ and $q_{R}$-integrable function, for $\alpha>0$, we have

$$
s t_{A}-\lim _{n}\left\|K_{n}^{q_{1, n}, q_{2, n}} f-f\right\|_{\omega_{2}}=0 .
$$

Proof Let $\tilde{K}_{n}^{q_{1, n}, q_{2, n}}$ be defined as

$$
\tilde{K}_{n}^{q_{1, n}, q_{2, n}}(f ; x, y)= \begin{cases}K_{n}^{q_{1, n}, q_{2, n}}(f ; x, y), & 0 \leq x<\frac{q_{1, n}}{1-q_{1, n}^{n}}, 0 \leq y<\frac{q_{2, n}}{1-q_{2, n}^{n}} \\ f(x, y), & x \geq \frac{q_{1, n}}{1-q_{1, n}^{n}}, y \geq \frac{q_{2, n}}{1-q_{2, n}^{n}} .\end{cases}
$$

From Lemma 1 , since $\left|K_{n}^{q_{1, n}, q_{2, n}}\left(1+t^{2}+s^{2} ; x, y\right)\right| \leq c\left(1+x^{2}+y^{2}\right)^{1+\alpha}$ for $x \in\left[0, \frac{q_{1, n}}{1-q_{1, n}^{n}}\right)$ and $y \in\left[0, \frac{q_{2, n}}{1-q_{2, n}^{n}}\right),\left\{\tilde{K}_{n}^{q_{1, n}, q_{2, n}}(f ; \cdot)\right\}$ is a sequence of linear positive operators acting from $C_{\omega_{1}}\left(\mathbb{R}_{0}^{2}\right)$ to $B_{\omega_{2}}\left(\mathbb{R}_{0}^{2}\right)$.

From (i) of Lemma 1, it is clear that

$$
s t_{A}-\lim _{n}\left\|\tilde{K}_{n}^{q_{1, n}, q_{2, n}}\left(e_{00} ; \cdot\right)-e_{00}\right\|_{\omega_{1}}=0
$$

holds. By (ii) of Lemma 1, we get

$$
\begin{aligned}
& \sup _{0 \leq x<\frac{q_{1, n}}{1-q^{n}, 0 \leq y<\frac{q_{2, n}}{1-q^{n}}}} \frac{\left|K_{n}^{q_{1, n}, q_{2, n}}\left(e_{10} ; \cdot\right)-e_{10}\right|}{1+x^{2}+y^{2}} \\
& =\sup _{0 \leq x<\frac{q_{1, n}}{1-q_{1, n}^{n}, 0 \leq y<\frac{q_{2, n}}{1-q_{2, n}}}} \frac{\left|x+\frac{1}{[2]_{q_{1, n}}} \frac{1}{[n]_{1, n}}-x\right|}{1+x^{2}+y^{2}} \\
& =\sup _{0 \leq x<\frac{q_{1, n}}{1-q_{1, n}^{n}, 0 \leq y<\frac{q_{2, n}}{1-q_{2, n}^{n}}}} \frac{1}{1+x^{2}+y^{2}} \frac{1}{[2]_{q_{1, n}}} \frac{1}{[n]_{q_{1, n}}} \\
& =\frac{1}{[2]_{q_{1, n}}} \frac{1}{[n]_{q_{1, n}}} \text {. }
\end{aligned}
$$


Since $s t_{A^{-}} \lim _{n} \frac{1}{[n]_{q_{1, n}}}=0, s t_{A^{-}} \lim _{n}\left\|\tilde{K}_{n}^{q_{1, n}, q_{2, n}}\left(e_{10} ; \cdot\right)-e_{10}\right\|_{\omega_{1}}=0$. Similarly, since $s t_{A^{-}}$ $\lim _{n} \frac{1}{[n]_{q_{2, n}}}=0, s t_{A}-\lim _{n}\left\|\tilde{K}_{n}^{q_{1, n}, q_{2, n}}\left(e_{01} ; \cdot\right)-e_{01}\right\|_{\omega_{1}}=0$. Also, we have from (iv) of Lemma 1

$$
\begin{aligned}
& \sup _{0 \leq x<\frac{q_{1, n}}{1-q_{1, n}^{n}}, 0 \leq y<\frac{q_{2, n}}{1-q_{2, n}^{n}}} \frac{\left|K_{n}^{q_{1, n}, q_{2, n}}\left(e_{20} ; \cdot\right)-e_{20}\right|}{1+x^{2}+y^{2}} \\
& =\sup _{0 \leq x<\frac{q_{1, n}}{1-q_{1, n}^{n}}, 0 \leq y<\frac{q_{2, n}}{1-q_{2, n}^{n}}} \frac{\left|q_{1, n} x^{2}+\left(q_{1, n}+\frac{2}{[2]_{1, n}}\right) \frac{1}{[n]_{q_{1, n}}} x+\frac{1}{[3]_{q_{1, n}}} \frac{1}{[n]_{q_{1, n}}^{2}}-x^{2}\right|}{1+x^{2}+y^{2}} \\
& \leq\left(1-q_{1, n}\right)+\left(\frac{q_{1, n}}{2}+\frac{1}{[2]_{q_{1, n}}}\right) \frac{1}{[n]_{q_{1, n}}}+\frac{1}{[3]_{q_{1, n}}} \frac{1}{[n]_{q_{1, n}}^{2}} .
\end{aligned}
$$

So, we can write

$$
\left\|\tilde{K}_{n}^{q_{1, n}, q_{2, n}}\left(e_{20} ; \cdot\right)-e_{20}\right\|_{\omega_{1}} \leq\left(1-q_{1, n}\right)+\left(\frac{q_{1, n}}{2}+\frac{1}{[2]_{q_{1, n}}}\right) \frac{1}{[n]_{q_{1, n}}}+\frac{1}{[3]_{q_{1, n}}} \frac{1}{[n]_{q_{1, n}}^{2}}
$$

Since $s t_{A}-\lim _{n}\left(1-q_{1, n}\right)=0, s t_{A}-\lim _{n}\left(\frac{q_{1, n}}{2}+\frac{1}{[2]_{q_{1, n}}}\right) \frac{1}{[n]_{q_{1, n}}}=0$ and $s t_{A}-\lim _{n} \frac{1}{[3]_{q_{1, n}}} \frac{1}{[n]_{q_{1, n}}^{2}}=0$, for each $\varepsilon>0$, we define the following sets.

$$
\begin{array}{ll}
D:=\left\{k:\left\|\tilde{K}_{n}^{q_{1, n}, q_{2, n}}\left(e_{20} ; \cdot\right)-e_{2}\right\|_{\omega_{1}} \geq \varepsilon\right\}, & D_{1}:=\left\{k: 1-q_{1, k} \geq \frac{\varepsilon}{3}\right\}, \\
D_{2}:=\left\{k:\left(\frac{q_{1, k}}{2}+\frac{1}{[2]_{q_{1, k}}}\right) \frac{1}{[n]_{q_{1, k}}} \geq \frac{\varepsilon}{3}\right\}, \quad D_{3}:=\left\{k: \frac{1}{[3]_{q_{1, k}}} \frac{1}{[n]_{q_{1, k}}^{2}} \geq \frac{\varepsilon}{3}\right\} .
\end{array}
$$

By (3.2), it is clear that $D \subseteq D_{1} \cup D_{2} \cup D_{3}$, which implies that for all $n \in \mathbb{N}$,

$$
\sum_{k \in D} a_{n k} \leq \sum_{k \in D_{1}} a_{n k}+\sum_{k \in D_{2}} a_{n k}+\sum_{k \in D_{3}} a_{n k}
$$

Taking limit as $n \rightarrow \infty$, we have

$$
s t_{A^{-}} \lim _{n}\left\|\tilde{K}_{n}^{q_{1, n}, q_{2, n}}\left(e_{20} ; \cdot\right)-e_{20}\right\|_{\omega_{1}}=0
$$

Similarly, since $s t_{A}-\lim _{n}\left(1-q_{2, n}\right)=0, s t_{A}-\lim _{n}\left(\frac{q_{2, n}}{2}+\frac{1}{[2]_{q_{2, n}}}\right) \frac{1}{[n]_{q_{2, n}}}=0$ and $s t_{A}-\lim _{n} \frac{1}{[3]_{q_{2, n}}} \times$ $\frac{1}{[n]_{q_{2, n}}^{2}}=0$, we write $s t_{A}-\lim _{n}\left\|\tilde{K}_{n}^{q_{1, n}, q_{2, n}}\left(e_{02} ; \cdot\right)-e_{02}\right\|_{\omega_{1}}=0$. So, the proof is completed.

If we define the function $\varphi_{x, y}(t, s)=(t-x)^{2}+(s-y)^{2},(x, y) \in\left[0, \frac{q_{1}}{1-q_{1}^{n}}\right) \times\left[0, \frac{q_{2}}{1-q_{2}^{n}}\right)$, then by Lemma 1 one gets the following result

$$
\begin{aligned}
K_{n}^{q_{1}, q_{2}}\left(\varphi_{x, y}(t, s) ; x, y\right)= & \left(q_{1}-1\right) x^{2}+\left(q_{2}-1\right) y^{2}+\frac{q_{1}}{[n]_{q_{1}}} x+\frac{q_{2}}{[n]_{q_{2}}} y \\
& +\frac{1}{[3]_{q_{1}}} \frac{1}{[n]_{q_{1}}^{2}}+\frac{1}{[3]_{q_{2}}} \frac{1}{[n]_{q_{2}}^{2}} .
\end{aligned}
$$

We use the modulus of continuity $\omega(f, \delta)$ defined as follows:

$$
\omega(f, \delta):=\sup \left\{|f(t, s)-f(x, y)|:(t, s),(x, y) \in \mathbb{R}_{0}^{2} \text { and } \sqrt{(t-x)^{2}+(s-y)^{2}} \leq \delta\right\}
$$


where $\delta>0$ and $f \in C_{B}\left(\mathbb{R}_{0}^{2}\right)$ the space of all bounded and continuous functions on $\mathbb{R}_{0}^{2}$. Observe that, for all $f \in C_{B}\left(\mathbb{R}_{0}^{2}\right)$ and $\lambda, \delta>0$, we have

$$
\omega(f, \lambda \delta) \leq(1+[\lambda]) \omega(f, \delta)
$$

where $[\lambda]$ is defined to be the greatest integer less than or equal to $\lambda$.

By the definition of modulus of continuity, we have

$$
|f(t, s)-f(x, y)| \leq \omega\left(f, \sqrt{(t-x)^{2}+(s-y)^{2}}\right)
$$

and by (3.3), for any $\delta>0$,

$$
|f(t, s)-f(x, y)| \leq\left(1+\left[\frac{\sqrt{(t-x)^{2}+(s-y)^{2}}}{\delta}\right]\right) \omega(f, \delta),
$$

which implies that

$$
|f(t, s)-f(x, y)| \leq\left(1+\frac{(t-x)^{2}+(s-y)^{2}}{\delta^{2}}\right) \omega(f, \delta) .
$$

Using the linearity and positivity of the operators $K_{n}^{q_{1}, q_{2}}$, we get from (3.4) and $K_{n}^{q_{1}, q_{2}}\left(e_{00}\right.$; $x, y)=1$ that, for any $n \in \mathbb{N}$,

$$
\begin{aligned}
\left|K_{n}^{q_{1}, q_{2}}(f ; x, y)-f(x, y)\right| & \leq K_{n}^{q_{1}, q_{2}}(|f(t, s)-f(x, y)| ; x, y)+|f(x, y)|\left|K_{n}^{q_{1}, q_{2}}\left(e_{00} ; x, y\right)-e_{00}\right| \\
& \leq K_{n}^{q_{1}, q_{2}}\left(\left(1+\frac{(t-x)^{2}+(s-y)^{2}}{\delta^{2}}\right) \omega(f, \delta) ; x, y\right) \\
& =\left(1+\frac{1}{\delta^{2}} K_{n}^{q_{1}, q_{2}}\left(\varphi_{x, y}(t, s) ; x, y\right)\right) \omega(f, \delta) .
\end{aligned}
$$

Now, if we replace $q_{1, n}$ and $q_{2, n}$ by sequences $\left(q_{1, n}\right)$ and $\left(q_{2, n}\right)$ to be two sequences satisfying (3.1), and we take $\delta:=\delta_{n}(x, y)=\sqrt{K_{n}^{q_{1, n}, q_{2, n}}\left(\varphi_{x, y}(t, s) ; x, y\right)}, 0 \leq x<\frac{q_{1, n}}{1-q_{1, n}^{n}}, 0 \leq y<\frac{q_{2, n}}{1-q_{2, n}^{n}}$, then we can write

$$
\left|K_{n}^{q_{1}, q_{2}}(f ; x, y)-f(x, y)\right| \leq 2 \omega(f, \delta) .
$$

\section{Competing interests}

The author did not provide this information.

\section{Acknowledgements}

The author is grateful to the referees for their useful remarks.

Received: 14 December 2012 Accepted: 1 July 2013 Published: 16 July 2013

\section{References}

1. Gupta, V, Vasishtha, V, Gupta, MK: Rate of convergence of the Szász-Kantorovich-Bezier operators for bounded variation functions. Publ. Inst. Math. (Belgr.) 72, 137-143 (2006)

2. Aral, A, Gupta, V: The q-derivative and application to q-Szász-Mirakyan operators. Calcolo 43, 151-170 (2006)

3. Mahmudov, NI: On q-parametric Szász-Mirakjan operators. Mediterr. J. Math. 7(3), 297-311 (2010)

4. Agratini, O, Dogru, O: Weighted statistical approximation by q-Szász type operators that preserve some test functions. Taiwan. J. Math. 14(4), 1283-1296 (2010) 
5. Derriennic, M: Modified Bernstein polynomials and Jacobi polynomials in q-calculus. Rend. Circ. Mat. Palermo Suppl. 76, 269-290 (2005)

6. Gupta, V: Some approximation properties of q-Durrmeyer operators. Appl. Math. Comput. 197, 172-178 (2008)

7. Gupta, V, Radu, C: Statistical approximation properties of q-Baskokov-Kantorovich operators. Cent. Eur. J. Math. 7(4), 809-818 (2009)

8. Mahmudov, NI: Statistical approximation properties of Baskokov and Baskokov-Kantorovich operators based on the q-integers. Cent. Eur. J. Math. 8(4), 816-826 (2010)

9. Aral, A, Gupta, V: Generalized Szász Durrmeyer operators. Lobachevskii J. Math. 32(1), 23-31 (2011)

10. Örkcü, M, Doğru, O: Statistical approximation of a kind of Kantorovich type $q$-Szász-Mirakjan operators. Nonlinear Anal. 75(5), 2874-2882 (2012)

11. Mursaleen, M, Khan, A, Srivastava, HM, Nisar, KS: Operators constructed by means of $q$-Lagrange polynomials and A-statistical approximation. Appl. Math. Comput. 219, 6911-6918 (2013)

12. Agrawal, PN, Gupta, V, Sathish Kumar, A: On q-analogue of Bernstein-Schurer-Stancu operators. Appl. Math. Comput. 219(14), 7754-7764 (2013)

13. Stancu, DD: A new class of uniform approximating polynomial operators in two and several variables. In: Alexits, G, Stechkin, SB (eds.) Proceedings of the Conference on Constructive Theory of Functions, pp. 443-455. Akadémiai Kiadó, Budapest (1972)

14. Barbosu, D: Some generalized bivariate Bernstein operators. Math. Notes 1, 3-10 (2000)

15. Erkuş, E, Duman, O: A-Statistical extension of the Korovkin type approximation theorem. Proc. Indian Acad. Sci. Math. Sci. 115(4), 499-507 (2003)

16. Erkuş, E, Duman, O: A Korovkin type approximation theorem in statistical sense. Studia Sci. Math. Hung. 43(3), 285-294 (2006)

17. Aktuğlu, H, Özarslan, MA, Duman, O: Matrix summability methods on the approximation of multivariate $q$-MKZ operators. Bull. Malays. Math. Sci. Soc. 34(3), 465-474 (2011)

18. Gasper, G, Rahman, M: Basic Hypergeometric Series. Cambridge University Press, Cambridge (1990)

19. Andrews, GE, Askey, R, Roy, R: Special Functions. Cambridge University Press, Cambridge (1999)

20. Marinkovic, S, Rajkovich, P, Stankovich, M: The inequalities for some types of $q$-integers. Comput. Math. Appl. 56 2490-2498 (2008)

21. Volkov, VI: On the convergence sequences of linear positive operators in the space of continuous functions of two variables. Dokl. Akad. Nauk SSSR 115, 17-19 (1957) (Russian)

22. Gadjiev, AD: Positive linear operators in weighted spaces of functions of several variables. Izv. Akad. Nauk Azerb. SSR, Ser. Fiz.-Teh. Mat. Nauk 4, 32-37 (1980)

23. Hardy, GH Divergent Series. Oxford University Press, London (1949)

24. Freedman, AR, Sember, JJ: Densities and summability. Pac. J. Math. 95, 293-305 (1981)

25. Fast, H: Sur la convergence statistique. Colloq. Math. 2, 241-244 (1951)

26. Cao, F, Liu, Y: Approximation theorems by positive linear operators in weighted spaces. Positivity 15, 87-103 (2011)

doi:10.1186/1029-242X-2013-324

Cite this article as: Örkcü: Approximation properties of bivariate extension of $q$-Szász-Mirakjan-Kantorovich operators. Journal of Inequalities and Applications 2013 2013:324.

\section{Submit your manuscript to a SpringerOpen ${ }^{\circ}$ journal and benefit from:}

- Convenient online submission

- Rigorous peer review

- Immediate publication on acceptance

- Open access: articles freely available online

- High visibility within the field

- Retaining the copyright to your article 